\title{
Separation and characterization of natural and synthetic macromolecules
}

\author{
Albena Lederer • Peter J. Schoenmakers
}

Published online: 5 October 2013

(C) Springer-Verlag Berlin Heidelberg 2013

This topical collection is dedicated to the separation and characterization of natural and synthetic macromolecules. The international symposia on separation and characterization of natural and synthetic macromolecules, abbreviated to SCM, were born in 2003 with a series of conferences organized in Amsterdam, and very quickly a lively international community developed with a main emphasis on separation techniques for macromolecules. The area of interest expanded with time to include complementary techniques for characterization of macromolecules, which makes powerful analysis of macromolecular systems possible. The idea to collect representative contributions in a special issue of Analytical and Bioanalytical Chemistry arose during the planning of the first SCM held in Dresden, in 2013. Today, SCM is a platform for discussion and collaboration among scientists with different scientific backgrounds interested in separation techniques and characterization methods for macromolecules and it represents a forum of experts in chemical analysis, polymer research and the life sciences.

Macromolecules, independent of their origin, synthetic or natural, are essential in our life. Complex polymer architectures with tailored designs are increasingly used in highpriority areas, such as microelectronics and medicine. Along with synthetic strategies, the control and analysis of their properties are of great significance for understanding the

Published in the topical collection Separation and Characterization of Natural and Synthetic Macromolecules with guest editors Albena Lederer and Peter J. Schoenmakers.

A. Lederer

Leibniz-Institut für Polymerforschung Dresden e.V., Hohe Str. 6, 01069 Dresden, Germany

\author{
A. Lederer $(\bowtie)$ \\ Technische Universität Dresden, 01062 Dresden, Germany \\ e-mail: lederer@ipfdd.de \\ P. J. Schoenmakers \\ Van 't Hoff Institute for Molecular Sciences, University of \\ Amsterdam, PO Box 94720, 1090 GE Amsterdam, The Netherlands \\ e-mail: p.j.schoenmakers@uva.nl
}

structure-property relationships. In this context, the need for accurate, reliable and efficient characterization methods is obvious. For effective and successful research in polymer separation science, interdisciplinarity is essential.

SCM provides a platform for promising characterization techniques in an early stage of their development, but also for hot topics related to synthetic and natural polymers on a fundamental or application-oriented level. This is reflected in this special issue. It starts with a Trends article and a Critical Review followed by Research Papers covering subjects such as human health, smart materials, and polymers/oligomers for food, cosmetics, additives, etc. The macromolecular systems discussed in this collection of articles are of synthetic or natural origin, ranging from glucose polymers, substituted cellulose ethers, Diels-Alder polymers with dynamic bonding, branched polymers, block copolymers, smart polymer brushes and biopolymers all the way to peptide surfaces.

We place strong emphasis on the development and improvement of separation methods, for example temperaturedependent size-exclusion chromatography and the coupling of different separation techniques to solve special problems, such as in situ reaction kinetics or structure elucidation of olefin copolymers. The separation techniques used in polymer science are nowadays far more diverse than size-exclusion chromatography. Techniques such as liquid adsorption chromatography, capillary electrophoresis (under critical conditions), hydrodynamic chromatography, field-flow fractionation (in all its variations) and the well-known temperature-rising elution fractionation are creatively used for solving specific issues. In combination with new detection techniques, such as light scattering and electrospray ionization mass spectrometry, and with complementary spectroscopic, microscopic and thermal analysis, comprehensive state-of the-art investigations are performed.

Apart from the latest developments in polymer separations, this issue features a contribution on polymer characterization at surfaces using time-of-flight secondary ion mass spectrometry, $\mathrm{X}$-ray atomic force microscopy and in situ spectroscopic ellipsometry. 


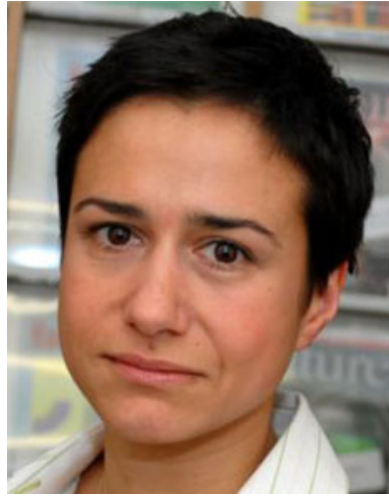

Albena Lederer is Head of the Polymer Separation Group at the Leibniz Institute of Polymer Research in Dresden and teaches as a Privatdozent at the Technische Universität Dresden. Her research interests are in the field of polymer analysis in general, with special focus on separation and scattering techniques. Her current activities are related to the structural aspects of dendritic polymers, novel separation techniques for branched polymers, intermolecular interactions of biofunctional polymers and in situ characterization of dynamic polymer bonding.

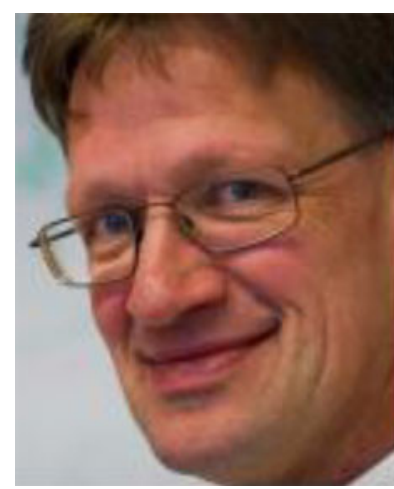

Peter J. Schoenmakers is a full Professor at the University of Amsterdam and a Group Leader at the Van 't Hoff Institute for Molecular Sciences. His group uses and develops advanced oneand two-dimensional separation techniques, electromigration techniques and field-flow fractionation. In the focus of his research are developments in mass spectrometry and two-dimensional liquid-chromatographic separations for generating novel insights into the structure and function of natural and synthetic macromolecules. His recent research interests include advanced chip-based separation systems based on nanotechnology. 